\title{
Uso de terapias não convencionais no manejo da crise aguda de asma refratária*
}

\author{
RAquel Hermes Rosa Oliveira ${ }^{1}$, AleXANDRe De Oliveira Ribeiro², \\ Gustavo Adolpho Junqueira Amarante ${ }^{2}$, Miguel Lia TedDE ${ }^{3}$
}

Uma paciente em crise aguda de asma, refratária ao uso de $\beta_{2}$-agonista inalatório e intravenoso, aminofilina intravenosa e corticóide, em ventilação mecânica, foi tratada com métodos terapêuticos não convencionais: broncoscopia, lavado broncoalveolar com $\mathrm{N}$-acetilcisteína e ventilação com halotano. Houve melhora dos parâmetros ventilatórios após o lavado e a resolução do broncoespasmo ocorreu após a anestesia, propiciando a extubação e alta da UTI. É feita uma revisão da literatura sobre o uso desses métodos na crise aguda de asma. (J Pneumol 2002;28(5):277-80)

\section{Non-conventional therapies to manage refractory acute asthma attack}

A patient with an acute asthma attack refractory to inhaled and intravenous $\beta_{2}$-agonist, aminophylline and corticosteroids was submitted to mechanical ventilation and treated with nonconventional therapies: bronchoscopy with bronchoalveolar lavage using $N$-acetylcysteine and halothane inhalation. The ventilatory parameters improved after lavage, however, bronchospasm resolution occurred only after anesthesia which was followed by extubation and discharge from the ICU. A review of the use of these non-conventional therapeutic modalities for the management of acute asthma attack is presented.

Descritores - Asma. Broncoscopia. Respiração artificial. Halotano. Espasmo brônquico.

Key words - Asthma. Bronchoscopy. Artificial respiration. Halothane. Bronchospasm.

O tratamento convencional da crise aguda de asma, de acordo com o II Consenso Brasileiro no Manejo da Asma de 1998 , consiste no uso de $\beta_{2}$-agonista inalatório e corticóide, podendo-se acrescentar brometo de ipratrópio e aminofilina, broncodilatador e corticóide por via intravenosa quando não há resposta à abordagem inicial ${ }^{(1)}$. Atualmente, apenas pequena percentagem dos casos de crise aguda de asma requer ventilação mecânica e, destes, aproximadamente $40 \%$ são extubados dentro de 24 horas e

\footnotetext{
* Trabalho realizado na Unidade de Terapia Intensiva do Hospital Sírio Libanês, São Paulo, SP.

1. Médica Pneumologista e Residente de Terapia Intensiva.

2. Médico da Unidade de Terapia Intensiva.

3. Cirurgião Torácico e Broncoscopista.

Endereço para correspondência - Raquel Hermes Rosa Oliveira, Hospital Sírio Libanês UTI-C, 1ํandar, Rua Dona Adma Jafet, 91 - 01308050 - São Paulo, SP. Tel. (11) 3155-0391; fax (11) 258-0876; e-mail: raquelhermes@ig.com.br
}

Recebido para publicação em 7/12/01. Aprovado, após revisão, em 30/7/02.
Siglas e abreviaturas utilizadas neste trabalho

UTI - Unidade de terapia intensiva

PEEP - Pressão positiva expiratória final

$\mathrm{FiO}_{2}$ - Fração inspirada de oxigênio

$\mathrm{ETCO}_{2}$ - Dióxido de carbono expirado

Pimax - Pressão inspiratória máxima em vias aéreas

FR - Freqüência respiratória

VC - Volume corrente

$\mathrm{PaO}_{2}$ - Pressão parcial arterial de oxigênio

$\mathrm{PaCO}_{2}$ - Pressão parcial arterial de gás carbônico

Rel I:E - Relação entre os tempos inspiratório e expiratório

$70 \%$ em 72 horas $^{(2)}$. No entanto, a manutenção do broncoespasmo, apesar do tratamento farmacológico, pode prolongar a ventilação mecânica, causar altas pressões em vias aéreas e maior risco de barotrauma. Em algumas dessas situações, podemos lançar mão de métodos não convencionais para tratamento do broncoespasmo, como o uso de agentes anestésicos voláteis e lavado brônquico por broncoscopia para retirada de tampões de secreção ${ }^{(3-}$ 13).

O objetivo deste trabalho é relatar um caso de crise aguda de asma em adulto que necessitou de ventilação mecânica e foi submetido a broncoscopia, lavado brônquico com $\mathrm{N}$-acetilcisteína e ventilação com halotano na unidade de terapia intensiva. 


\section{Relato Do CAso}

Mulher de 47 anos, asmática havia 20 anos, em uso de corticóide inalatório, teofilina e broncodilatador de demanda, deu entrada no pronto atendimento com crise aguda de asma havia um dia e piora havia quatro horas, a despeito do uso da medicação. A última crise havia sido há aproximadamente seis meses e, havia 10 anos, apresentou crise aguda grave, necessitando de ventilação mecânica por sete dias. Apresenta também depressão controlada com clomipramina. Não apresentou resposta ao tratamento hospitalar inicial, composto de fenoterol em inalação (três doses), 60mg de metilprednisolona e $240 \mathrm{mg}$ de aminofilina intravenosa. Evoluiu com fadiga muscular, diminuição do nível de consciência e acidose respiratória, sendo transferida para a UTI.

Ao exame físico na UTI, apresentava-se ansiosa, taquicárdica (120bpm), com PA de 155 x 70mmHg, freqüência respiratória de 30 incursões por minuto, utilizando musculatura acessória, sibilos intensos e difusos à ausculta. Sua radiografia de tórax mostrava um padrão de hiperinsuflação sem opacidades parenquimatosas. O tratamento instituído consistiu de metilprednisolona $160 \mathrm{mg}$ ao dia, aminofilina em infusão contínua $0,6 \mathrm{mg} / \mathrm{kg} / \mathrm{h}$, terbutalina $0,25 \mathrm{mg}$ subcutânea, aerossol de brometo de ipratrópio associado a fenoterol (seis puffs a cada duas horas) e claritromicina $500 \mathrm{mg}$ a cada 12 horas. Houve progressão do quadro, sendo realizada intubação orotraqueal com cânula número 8 com cuff de baixa pressão. A paciente foi mantida sob sedação contínua com midazolam e fentanil e ventilada em um aparelho Bird 8400 em regime de pressão controlada, com freqüência de 10 ciclos por minuto, PEEP aproximadamente $80 \%$ da auto-PEEP medida no ventilador e $\mathrm{FiO}_{2}$ de 40\%. A Tabela 1 contém os parâmetros ventilatórios durante a internação. No dia seguinte, a paciente evoluiu com picos febris $\left(38,4^{\circ} \mathrm{C}\right)$,

TABELA 1

Parâmetros ventilatórios durante a internação

\begin{tabular}{lccrrrrrr}
\hline & D1 & AB1 & DB1 & AB2 & DB2 & AH & H & AE \\
\hline FiO $_{2}$ & 40 & 30 & 30 & 35 & 35 & 30 & 30 & 30 \\
Pimax & 34 & 33 & 22 & 26 & 18 & 21 & 18 & 17 \\
PEEP & 10 & 15 & 6 & 10 & 6 & 9 & 6 & 5 \\
Auto-PEEP & 12 & 17 & 8 & 12 & 8 & 11 & 7 & - \\
FR & 10 & 12 & 12 & 12 & 10 & 10 & 12 & 12 \\
VC & 400 & 300 & 500 & 380 & 480 & 400 & 540 & 500 \\
$\mathrm{PaO}_{2}$ & 84,3 & 86,0 & 91,2 & 88,0 & 114,0 & 87,3 & 100,0 & 103,0 \\
$\mathrm{PaCO}_{2}$ & 59,4 & 76,6 & 53,0 & 66,8 & 50,8 & 51,5 & 50,0 & 36,6 \\
$\mathrm{ETCO}_{2}$ & - & 80 & 56 & 70 & 52 & 55 & 52 & 40
\end{tabular}

$\mathrm{D} 1$ = primeiro dia de internação, $\mathrm{AB} 1$ = antes da primeira broncoscopia, $\mathrm{DB} 1=$ depois da primeira broncoscopia, $\mathrm{AB} 2$ = antes da segunda broncoscopia, $\mathrm{DB} 2=$ depois da segunda broncoscopia, $\mathrm{AH}=$ antes do uso de halotano, $\mathrm{H}=$ durante o uso de halotano, $\mathrm{AE}=$ antes da extubação. persistência do broncoespasmo e dificuldade ventilatória, mantendo $\mathrm{ETCO}_{2}$ em torno de $70 \mathrm{mmHg}$. A terbutalina passou a ser administrada por via intravenosa contínua na dose de $5 \mathrm{mcg} / \mathrm{min}$, introduziu-se ceftriaxona e utilizou-se pancurônio em infusão contínua $(0,04 \mathrm{mcg} / \mathrm{kg} / \mathrm{min})$ para melhor ajuste da ventilação.

No terceiro dia, devido à progressão do broncoespasmo, a paciente foi submetida a broncoscopia com lavado brônquico utilizando-se um broncoscópio Olympus BF tipo P20D com 4,9mm de diâmetro externo. Durante o procedimento, a $\mathrm{FiO}_{2}$ foi aumentada para $100 \%$ e os demais parâmetros ventilatórios foram mantidos. Em cada segmento pulmonar, foram instilados e aspirados de 15 a $20 \mathrm{ml}$ de solução fisiológica aquecida. Houve retorno de grande quantidade de secreção mucóide espessa, principalmente dos lobos superiores, e melhora imediata da ventilação com aumento do volume corrente e queda da $\mathrm{PaCO}_{2}$ de 77 para $53 \mathrm{mmHg}$, que se manteve até o dia seguinte, quando foi retirado o curare (Tabela 1). Após 12 horas sem curare, a paciente voltou a apresentar piora progressiva, queda do volume corrente e aumento da $\mathrm{PaCO}_{2}$ para $66,8 \mathrm{mmHg}$, a despeito do aumento da dose dos broncodilatadores, do uso de sulfato de magnésio e da reintrodução do pancurônio. Assim, no quinto dia, foi submetida a nova broncoscopia associada a lavado brônquico com N-acetilcisteína a $20 \%$ (30ml) diluída em $60 \mathrm{ml}$ de solução fisiológica aquecida. Foi pré-medicada com doses extras de $\beta_{2}$-agonista inalatório devido ao efeito irritante da $\mathrm{N}$-acetilcisteína sobre as vias aéreas e instiladas alíquotas de aproximadamente $3 \mathrm{ml}$ da solução em cada segmento do pulmão direito; após um intervalo de 15 minutos, foi aspirada grande quantidade de secreção mucóide espessa. $\mathrm{O}$ procedimento foi repetido à esquerda com utilização de doses adicionais de $\beta_{2}$-agonista e ventilação com ambu devido ao baixo volume corrente obtido com a ventilação mecânica.

Como houve melhora dos parâmetros ventilatórios por apenas oito horas, foi iniciada ventilação com o anestésico halotano na concentração de 1,5\% no ventilador Servo 900. Após uma hora de anestesia, cessaram os sibilos, houve aumento do volume corrente, queda da $\mathrm{PaCO}_{2}$ para $50 \mathrm{mmHg} e$ a dose da terbutalina foi reduzida para a metade. A anestesia perdurou por 36 horas, com melhora progressiva. A paciente foi extubada no $9^{\circ}$ dia, 48 horas após o término do uso do halotano. Os resultados das culturas dos lavados brônquicos e hemoculturas foram negativos. A Tabela 2 contém o resumo da internação. O Gráfico 1 ilustra a evolução da auto-PEEP durante a internação com queda dos valores depois da realização dos lavados brônquicos e do uso do anestésico inalatório (DB1, DB2 e H). O Gráfico 2 mostra a evolução da $\mathrm{PaCO}_{2}$ com estabilização dos valores depois do segundo lavado (DB2). 
TABELA 2

Resumo da internação na UTI

D1 IOT e sedação contínua. Terbutalina IV, fenoterol + BI inalatório, aminofilina $0,6 \mathrm{mg} / \mathrm{kg} / \mathrm{h}$, metilprednisolona $160 \mathrm{mg} /$ dia, claritromicina

D2 Terbutalina $0,5 \mathrm{mcg} / \mathrm{kg} / \mathrm{min}$, pancurônio $0,04 \mathrm{mcg} / \mathrm{kg} / \mathrm{min}$, sulfato de magnésio, ceftriaxona

\begin{tabular}{ll}
\hline D3 & Lavado brônquico com SF a 0,9\% \\
\hline D4 & Mantido \\
\hline D5 & Lavado brônquico com N-acetilcisteína \\
\hline D6 & Halotano a 1,5\% \\
\hline D7 & Redução das doses de terbutalina e aminofilina \\
\hline D8 & Suspenso halotano \\
\hline D9 & Extubação \\
\hline D11 & Alta da UTI \\
\hline
\end{tabular}

IOT = intubação orotraqueal; $\mathrm{BI}=$ brometo de ipratrópio.

Como complicações, apresentou fraqueza muscular generalizada e hipertensão arterial sistêmica, ambas revertidas após redução da dose do corticóide.

\section{DISCUSSÃO}

A hipersecreção de muco com preenchimento de brônquios e bronquíolos é um dos mecanismos de obstrução ao fluxo aéreo na asma. Sabe-se que a impactação de material mucóide proteináceo nas vias aéreas contribui na patogênese da crise aguda prolongada (status asmaticus) e provavelmente reflete a ação de mediadores inflamatórios incluindo prostaglandinas, leucotrienos e histamina, que aumentam a produção de muco. Há evidência ainda de retardo do clearance mucociliar na asma. Estudos de necropsia de pacientes que morreram em crise asmática grave revelaram brônquios e bronquíolos preenchidos por muco viscoso que, em alguns casos, se estendia até a traquéia ${ }^{(2)}$. Esses dados corroboram a associação entre crises agudas refratárias e a presença de muco na árvore brônquica.

O uso de lavado broncoalveolar tem sido descrito desde $1966^{(3)}$ como terapia mucolítica adjuvante na crise de asma e em várias outras entidades, como fibrose cística, doença pulmonar obstrutiva crônica, bronquiectasias, proteinose alveolar, pneumonite química, etc. Há vários relatos na literatura do seu uso em crises de asma prolongadas devido ao excesso de muco. A fim de aumentar o clearance de muco, foram adicionados agentes mucolíticos como a $\mathrm{N}$-acetilcisteína, que diminui o volume e a viscosidade do muco por afetar diretamente a produção

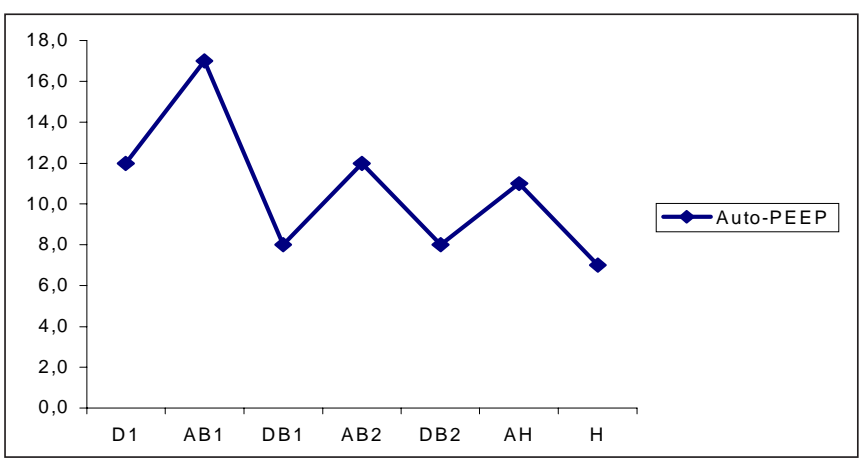

Gráfico 1 - Evolução da auto-PEEP. D1 = primeiro dia de internação, $A B 1=$ antes da primeira broncoscopia, DB1 = depois da primeira broncoscopia, $A B 2=$ antes da segunda broncoscopia, $D B 2=$ depois da segunda broncoscopia, $\mathrm{AH}=$ antes do uso de halotano, $\mathrm{H}=$ durante $\mathrm{o}$ uso de halotano.

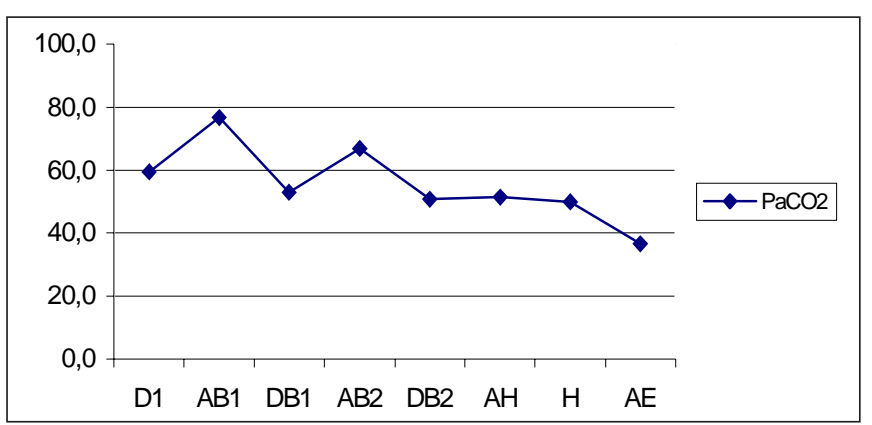

Gráfico 2 - Evolução da $\mathrm{PaCO}_{2}$. D1 = primeiro dia de internação, $A B 1=$ antes da primeira broncoscopia, $D B 1=$ depois da primeira broncoscopia, $A B 2=$ antes da segunda broncoscopia, $D B 2=$ depois da segunda broncoscopia, $\mathrm{AH}=$ antes do uso de halotano, $\mathrm{H}$ = durante $o$ uso de halotano, $A E=$ antes da extubação.

da glicoproteína. Como esse agente possui efeito irritativo em contato com a mucosa brônquica, há risco de exacerbação do broncoespasmo; assim, agentes $\beta_{2}$-agonistas e corticóides têm sido adicionados à solução de $\mathrm{N}$-acetilcisteína, tanto para realização de lavado brônquico como para nebulização. Um único lavado pode não ser eficaz para remover toda a secreção. Shridharani $e$ Maxson relataram o caso de uma paciente alérgica à aminofilina tratada com lavado brônquico com $30 \mathrm{ml}$ de $\mathrm{N}$-acetilcisteína, $\beta_{2}$-agonista e $125 \mathrm{mg}$ de metilprednisolona; houve recuperação da ventilação após o segundo lavado, com extubação em 24 horas(4). Millman et al. relataram um caso de crise refratária que necessitou de dois lavados com $\mathrm{N}$-acetilcisteína, $\beta_{2}$-agonista e corticóide ${ }^{(5)}$. Henke et al. relataram o caso de uma paciente de 22 anos que foi submetida a anestesia com isoflurano e ventilação com heliox (mistura de 60\% oxigênio e 40\% hélio), porém só obteve melhora da troca gasosa e da ventilação após lavado com $\mathrm{N}$-acetilcisteína ${ }^{(6)}$. Outro agente mucolítico com aparente benefício na asma é a DNase 
humana recombinante, cuja utilidade em pacientes com fibrose cística está bem estabelecida ${ }^{(7)}$. O lavado broncoalveolar tem sido cada vez mais utilizado em pacientes graves submetidos a ventilação mecânica com relativa segurança, desde que a hipoxemia, a instabilidade cardiovascular e os distúrbios hidroeletrolíticos sejam corrigidos antes do procedimento. As complicações mais comuns são broncoespasmo e hipoxemia leve, que são revertidos em até duas horas com o uso de broncodilatadores e aumento da oferta de oxigênio ${ }^{(8,9)}$. No caso apresentado, a despeito das precauções tomadas como o aumento da $\mathrm{FiO}_{2}$ e o uso de $\beta_{2}$-agonista antes do procedimento, houve dificuldade de ventilação e necessidade de insuflação manual com ambu durante a segunda broncoscopia, não havendo, porém, nenhuma complicação importante. Durante os lavados, houve saída de tampões de muco espesso, o que levou à melhora do broncoespasmo, permitindo melhor ventilação. Acreditamos que tal melhora seja devida à retirada dos tampões de muco, já que a paciente vinha em uso de $\beta_{2}$-agonista endovenoso contínuo e inalatório a cada duas horas havia mais de 48 horas. Entretanto, o benefício não foi mantido e optou-se por outra estratégia para manejo do broncoespasmo. Vale salientar que o emprego do lavado brônquico para manejo da crise aguda de asma não é preconizado de rotina por ausência de ensaios clínicos comprovando e quantificando seu benefício e pelo risco de piora do broncoespasmo, e, antes de optar por tal medida, devem-se excluir outras causas de manutenção do status asmaticus.

O halotano é um potente anestésico introduzido em 1956 pelo seu fácil manejo e rápido despertar após o uso. Seu efeito broncodilatador faz-se por bloqueio vagal e por ação direta sobre a musculatura lisa brônquica contraída, ou seja, em broncoespasmo, sendo oportuno na crise asmática ${ }^{(10)}$. Vários relatos de caso confirmam a utilidade desse anestésico no tratamento de crises refratárias ao tratamento clínico intensivo e, mais recentemente, Padkin et al. utilizaram ventilação com halotano para evitar a intubação de uma paciente de 41 anos em insuficiência respiratória aguda por crise asmática ${ }^{(11-13)}$. Outros agentes anestésicos voláteis, como isoflurano e sevoflurano, também são eficazes como broncodilatadores, sendo que o último causa menos complicações cardiovasculares que o halotano. Seus efeitos colaterais incluem hipocontratilidade miocárdica, hipotensão e vasodilatação periférica, que são dependentes da dose; taquiarritmias, que podem ser um problema em pacientes em uso de $\beta_{2}$-agonista e metilxantina endovenosos; hepatotoxicidade reversível após suspensão do uso, porém com risco de insuficiência hepática aguda em 1:35.000 $\operatorname{casos}^{(14)}$. No caso apresentado, a resolução da crise asmática evidencia o efeito broncodilatador do anestésico, uma vez que altas doses de $\beta_{2}$-agonista e corticóide por cinco dias não ha- viam controlado os sintomas e, só após o uso do halotano, houve melhora mantida da ventilação e da troca gasosa, como ilustrada nos Gráficos 1 e 2; 24 horas após o término da anestesia, com parâmetros ventilatórios normais, foi possível a extubação. A despeito de manter-se taquicárdica, com freqüência cardíaca de $140 \mathrm{bpm}$, a paciente não apresentou complicações importantes pelo uso do halotano.

O presente caso ilustra a possibilidade terapêutica de duas modalidades pouco utilizadas em nosso meio para manejo da crise aguda de asma e que devem ser lembradas nos casos refratários. No entanto, ainda há necessidade de estudos com um número suficiente de pacientes para definir as indicações e qual o subgrupo que se beneficiaria de ventilação com anestésico volátil e, principalmente, do uso de lavado brônquico para retirada de tampões de secreção.

\section{REFERÊNCIAS}

1. II Consenso Brasileiro no Manejo da Asma. J Pneumol 1998;24:173276.

2. Fraser RS, Colman N, Muller NL, Paré PD. Asthma. In:__. Frase and Paré's diagnosis of diseases of the chest. $4^{\text {th }}$ ed. Philadelphia: W.B. Saunders, 1999;2077-166.

3. Thompson HT, Pryor WJ, Hill L. Bronchial lavage in the treatment of obstructive lung disease. Thorax 1966;21:557-9.

4. Shridharani M, Maxson R. Pulmonary lavage in a patient in status asthmaticus receiving mechanical ventilation: a case report. Ann Allergy 1982;49:156-8.

5. Millman M, Goodman AH, Goldstein IM, Millman FM, Van Campen SS. Status asthmaticus: use of acetylcysteine during bronchoscopy and lavage to remove mucous plugs. Ann Allergy 1983;50:85-93.

6. Henke CA, Hertz M, Gustafson P. Combined bronchoscopy and mucolytic therapy for patients with severe refractory status asthmaticus on mechanical ventilation: a case report and review of the literature. Crit Care Med 1994;22:1880-3.

7. Durward A, Forte V, Shemie SD. Resolution of mucus plugging and atelectasis after intratracheal rhDNase therapy in a mechanically ventilated child with refractory status asthmaticus. Crit Care Med 2000; 28:560-2

8. Lang DM, Simon RA, Mathison DA, Timms RM, Stevenson DD. Safety and possible efficacy of fiberoptic bronchoscopy with lavage in the management of refractory asthma with mucous impactation. Ann Allergy 1991;67:324-30.

9. Djukanovic R, Wilson JW, Lai CK, Holgate ST, Howarth PH. The safety aspects of fiberoptic bronchoscopy, bronchoalveolar lavage, and endobronchial biopsy in asthma. Am Rev Respir Dis 1991;143:772-7.

10. Benumof JL. Respiratory physiology and respiratory function during anesthesia. In: Miller RD. Anesthesia. $3^{\text {rd }}$ ed. New York: Churchill Livingstone, 1990;505-50.

11. Padkin AJ, Baigel G, Morgan GA. Halothane treatment of severe asthma to avoid mechanical ventilation. Anaesthesia 1997;52:994-7.

12. Rosseel P, Lauwers LF, Baute L. Halothane treatment in life-threatening asthma. Int Care Med 1985;11:241-6.

13. O'Rourke PP, Crone RK. Halothane in status asthmaticus. Crit Care Med 1982;10:341-3.

14. Marshall BE, Longnecker DE. Anestésicos gerais. In: Hardman JG, Limbird LE. As bases farmacológicas da terapêutica. 9ª ed. Rio de Janeiro: McGraw-Hill Interamericana, 1996;226-40. 